\title{
Optical and Structural Properties of Emerging Dilute III-V Bismides
}

\author{
B. H. Bononi dos Santos ${ }^{a}$, Y Galvão Gobato ${ }^{a}$, and M. Henini ${ }^{\text {b* }}$ \\ ${ }^{a}$ Departamento de Física, Universidade Federal de São Carlos (UFSCAR) 13560-905, São Carlos, SP, Brazil \\ ${ }^{b}$ School of Physics and Astronomy, Nottingham Nanotechnology and Nanoscience Centre, \\ University of Nottingham, Nottingham NG7 2RD, U.K.
}

(Received September 15, 2014, Revised September 18, 2014, Accepted September 19, 2014)

\begin{abstract}
In this paper, we present a review of optical and structural studies of $\mathrm{GaBi}_{\mathrm{x}} \mathrm{As}_{1-\mathrm{x}}$ epilayers grown by Molecular Beam Epitaxy (MBE) on (311)B and (001) GaAs substrates with different As fluxes. The results indicate that under near-stoichiometric conditions the bismuth incorporation is higher for samples grown on (311)B GaAs substrates than for those grown on (001) GaAs. In addition, carrier localization effects in GaBiAs layers are clearly revealed for both samples by optical measurements. The (311)B samples showed evidence of higher density of defects. It has also been found that the nonradiative centers play a significant role in the recombination process in this material system. The influence of post-growth annealing on the microstructural, optical, and magneto-optical properties was also investigated. An important improvement of optical and spin properties after thermal annealing due to the reduction of defects in the GaBiAs layers was observed.
\end{abstract}

Keywords : Bismides, Molecular Beam Epitaxy, Defects, Growth, GaAsBi alloys

\section{Introduction}

Dilute III-V bismide materials have attracted much attention in recent years due to their unusual fundamental physical properties such as a large band gap reduction (about $85 \mathrm{meV}$ per percent of $\mathrm{Bi}$ ) and a strong enhancement of the spin orbit splitting due to incorporation of $\mathrm{Bi}$ in GaAs [1-21]. Owing to these remarkable properties, these materials hold promise for several applications in spintronics and for long-wavelength optoelectronics such as highly efficient and temperature stable semiconductor lasers. However, from a technological point of view, previous studies [1-21] showed that further optimization of the material growth conditions is necessary to obtain high quality properties required for technological applications.

GaAsBi alloys are usually grown at low temperatures $\left(270-400^{\circ} \mathrm{C}\right)$ in order to enhance the incorporation of $\mathrm{Bi}$ in GaAs [1]. For these growth conditions a high density of defects is usually incorporated, which results in localization of carriers and degradation of the optical efficiency. A thermal annealing process is usually employed to enhance the photoluminescence (PL) intensity of such materials. The effects of thermal annealing and identification of the mechanisms that affect the PL properties of GaBiAs are thus very important issues for future electronic, optoelectronic, and spintronic dilute bismide-based devices. In addition, it was previously

* [E-mail] Mohamed.Henini@Nottingham.ac.uk This paper was presented at IMCM 2014. 
reported that the incorporation of Bi results in an increase of the electron g-factor [15] and in a decrease of spin relaxation times due to spin-orbit interactions $[14,15]$. Such findings further indicate that GaBiAs is an interesting material for spintronics. Nevertheless, there have been few studies on the spin properties of GaBiAs layers and the effects of reduction of defects on thermal annealing and improvement of growth conditions on these properties [9-11]. Additional studies are thus necessary for improvement of material quality for optoeletronic and spintronics applications. Furthermore, the growth of epitaxial layers on high-index planes offers other possibilities to develop applications requiring modified properties with respect to devices grown on the conventional (001)-substrates. It was previously reported that growth on substrates with different orientations could influence the growth, impurity incorporation, spin and electronic properties, lasing performance, and piezoelectric effects.

In this work, we review the physical properties of $\mathrm{GaBi}_{x} \mathrm{As}_{1-\mathrm{x}}$ epilayers grown by molecular beam epitaxy (MBE) on (311)B and (001) GaAs substrates using different As fluxes. Photoluminescence (PL) and high resolution $\mathrm{X}$-ray diffraction (HRXRD) results indicate that under near-stoichiometric conditions the bismuth incorporation is enhanced for samples grown on (311)B relative to those grown on (001) GaAs substrates. The influence of post-growth annealing on the microstructural, PL, and spin properties is also investigated. It was observed that thermal annealing remarkably improves the optical and spin properties of GaBiAs layers.

\section{Structural Properties}

$\mathrm{GaBi}_{\mathrm{x}} \mathrm{As}_{1-\mathrm{x}}$ films were grown by $\mathrm{MBE}$ on semi-insulating (001) and (311)B GaAs substrates at a growth temperature of about $350^{\circ} \mathrm{C}$ with different $\mathrm{As}$ to $\mathrm{Ga}$ flux ratios. A growth rate of $1 \mu \mathrm{m} / \mathrm{h}$ was adopted for all samples. The nominal thickness of all epilayers is $1 \mu \mathrm{m}$. Details of the growth conditions of such materials are reported in Ref. [1].

The HRXRD results for GaBiAs epilayers grown under near-stoichiometric conditions on (001) and on (311) B GaAs substrates are shown in Figs. 1(a) and 1(b). Two peaks were observed and they are associated with the GaAs substrate and the GaBiAs layer [1]. The intensity of the GaBiAs layer grown on the (311) B substrates is lower than that grown on (100), which indicates that the former are thinner, likely due to a reduction of growth. TEM images (Figs. 1(c) and 1(d)) show evidence of extended structural defects in both epilayers. It was observed that, for (100) grown samples, the main structural defects are dislocations. The $\mathrm{Bi}$ concentrations were obtained from HRXRD results as a function of As flux used for the growth process for different (001) and (311)B epilayers (Fig. 2). The maximum Bi concentration was obtained near a stoichiometric condition [1]. In addition, higher Bi incorporation was observed for sam-

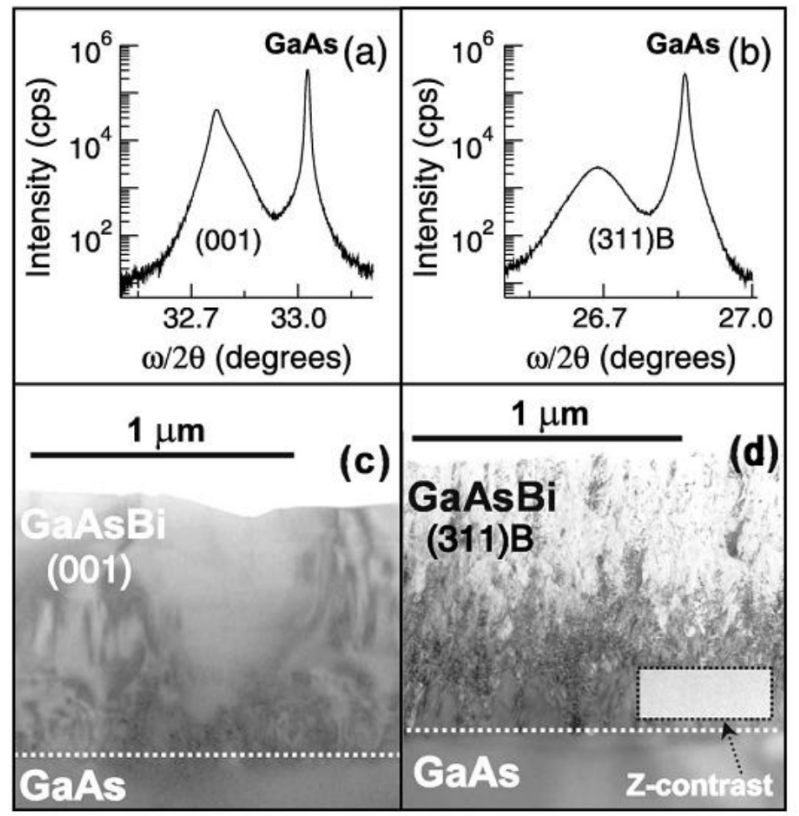

Figure 1. HRXRD and TEM results for GaBiAs layers grown on (001) and (311)B GaAs substrates [1]. 
ples grown on (311)B GaAs substrates. This results in narrower band gap energies, as will be discussed later.

The presence of structural defects in these layers contributes to degradation of the optical quality of such materials. In particular, the results show that extended defects are generated after the growth of a critical thickness of nearly defect-free GaAsBi, as revealed in the TEM image of the (311)B epilayer (Fig. 1(d)) [1]. It was suggested that after the growth of such critical thickness the defects propagate down to the interface and relax by plastic deformation. This effect is an important factor for the growth process, and could result in the formation of polycrystalline grains and other types of defects in the material. A higher density of defects closer to the surface was observed for the stoichiometric (311)B sample, and this may be related to its higher $\mathrm{Bi}$ amount. The structural properties of the GaBiAs layer therefore must be improved by further optimization of the growth conditions. In conclusion, enhancement of the Bi concentration for GaBiAs layers grown by $\mathrm{MBE}$ on (311)B GaAs substrates in comparison to the conventional (001) orientation was observed, and this led to

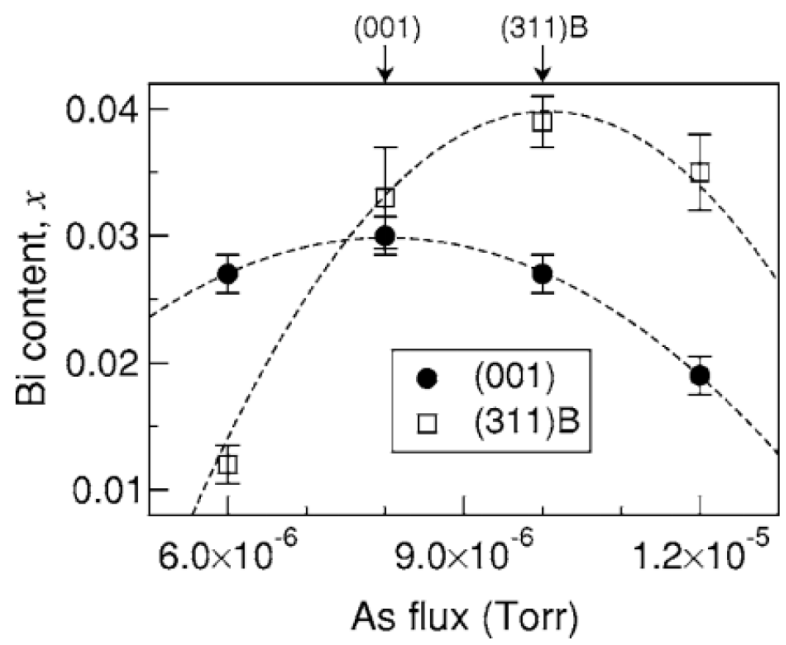

Figure 2. Bi composition as a function of the As flux used for the growth of GaBiAs layers on (001) and (311)B GaAs substrates [1]. reduced band gaps. The growth of GaBiAs alloys on (311)B GaAs substrates thus has significant potential for the development of GaAs based long-wavelength device applications.

\section{Optical Properties}

Optical properties of GaBiAs layers grown on both substrate orientations were investigated by using photomodulated transmittance (PT) and photoluminescence techniques (Figs. 3 and 4) at room temperature [5,7]. It was observed that the PT resonance for the layer grown on (311)B is at lower energy and broader than that for (001) GaAs layers [5]. These results are consistent with the finding of higher incorporation of $\mathrm{Bi}$ in samples grown on (311)B GaAs substrates [1]. The energy gap of the GaBiAs layers

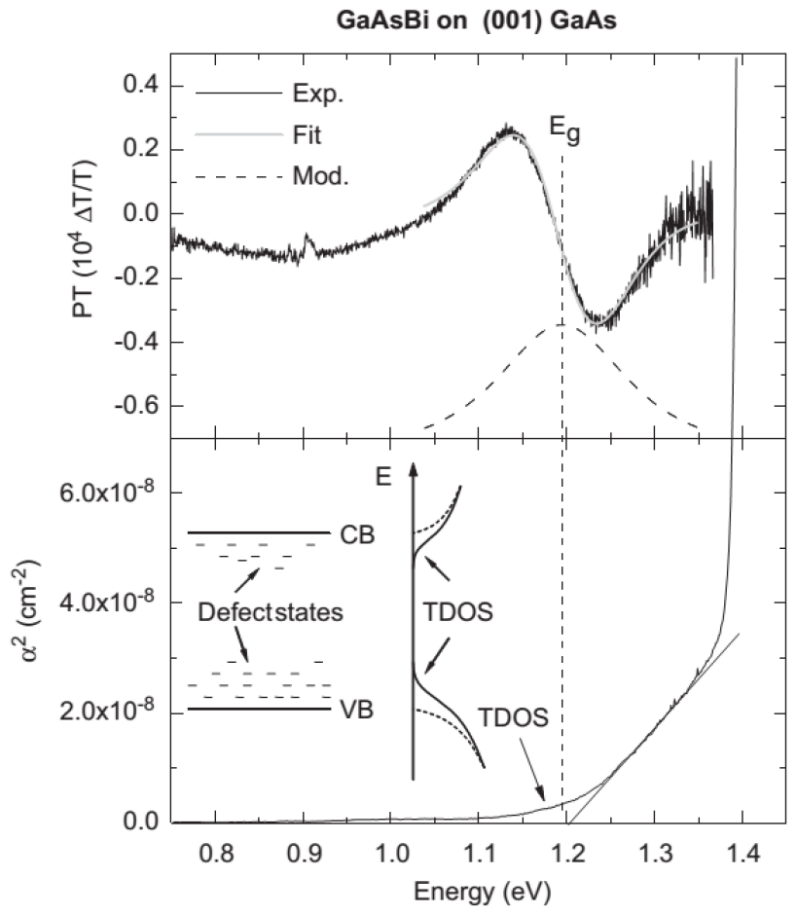

Figure 3. Photomodulated transmittance spectrum and the square absorption coefficient for stoichiometric GaBiAs layers grown on (001) GaAs substrate with As 2 flux of $8 \times 10^{-6}$ Torr [5] (Sample MS820). 


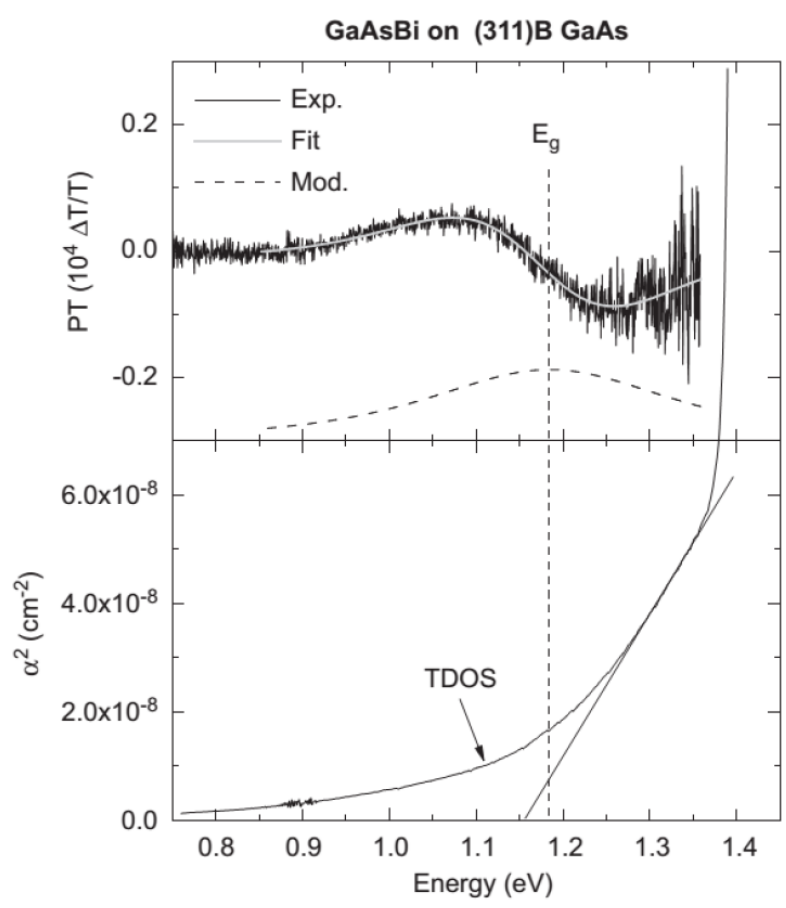

Figure 4. Photomodulated transmittance spectrum and the square absorption coefficient for GaBiAs layers grown on (311)B GaAs substrate with $A s_{2}$ flux of $8 \times 10^{-6}$ Torr [5] (Sample MS824).

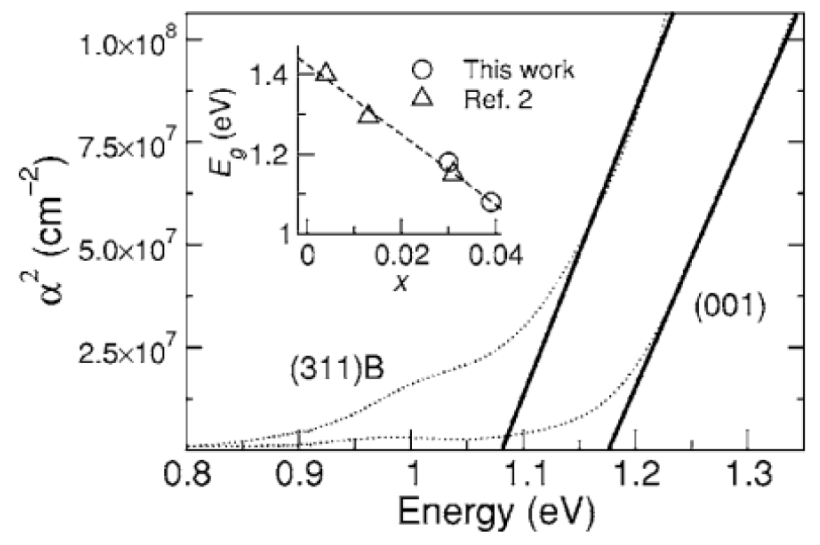

Figure 5. Square of the absorption coefficient for two stoichiometric GaBiAs layers grown on (001) and (311)B GaAs substrates (samples MS820 and MS825) [1].

was obtained from the square of the absorption coefficient $\alpha^{2}$ around the band-gap edge. A linear dependence with photon energy was obtained, consistent with the direct bad gap nature of GaBiAs material. Deviations from linear behaviour were ob-

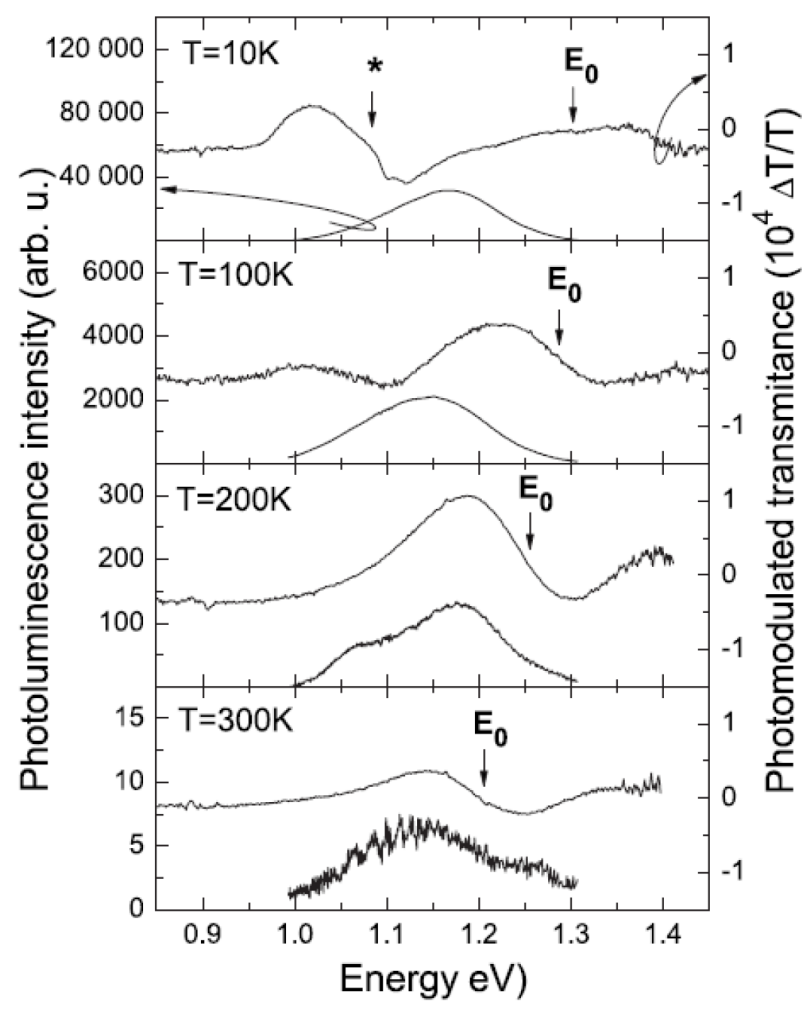

Figure 6. Photomodulated transmittance and photoluminescence spectra for different temperatures [7].

served for both the (001) and (311)B samples and were associated with the tail of density of states (TDOS) in GaBiAs, which are due to defect states in the band gap region. The energy gap obtained from these measurements is about $1.2 \mathrm{eV}$ for the sample grown on the (001) GaAs substrate and about $1.15 \mathrm{eV}$ for that grown on the (311)B substrate.

For the GaBiAs layer grown with a stoichiometric condition on a (311)B GaAs substrate, the energy gap is about $90 \mathrm{meV}$ lower than that for the GaBiAs (001) layer due to higher Bi incorporation (Fig. 5). These results are corroborated by $\mathrm{PL}$ measurements in the same samples.

In the following, we focus on a study of localization effects on stoichiometric GaBiAs layers grown on a (001) GaAs substrate. These effects were investigated by measuring photomodulated transmittance (PT), photoluminescence (PL), and time-resolved PL 
[7]. The band gap related transition is clearly observed in the PT spectrum for different temperatures (Fig. 6). At $10 \mathrm{~K}$ the band gap related absorption is almost not visible in the PT spectrum while another spectral feature that is not yet understood was observed at around $1.1 \mathrm{eV}$. The temperature dependence of the PL spectrum shows a strong decrease of the signal below 180K [7], which indicates carrier localization in these GaBiAs layers. The energy gap was determined from PT results and they are illustrated in Fig. 7. The effect of carrier localization in this alloy is evidenced by the observed large Stokes shift (120 meV at low temperatures and $40 \mathrm{meV}$ at room temperature) between the energy gap, the PT transition, and the PL energy peak. For intermediate temperatures, two PL bands are observed and they are associated with the localization of carriers for defects of different nature, which accounts for the observation of different PL bands with different activation energies. The presence of these two emission

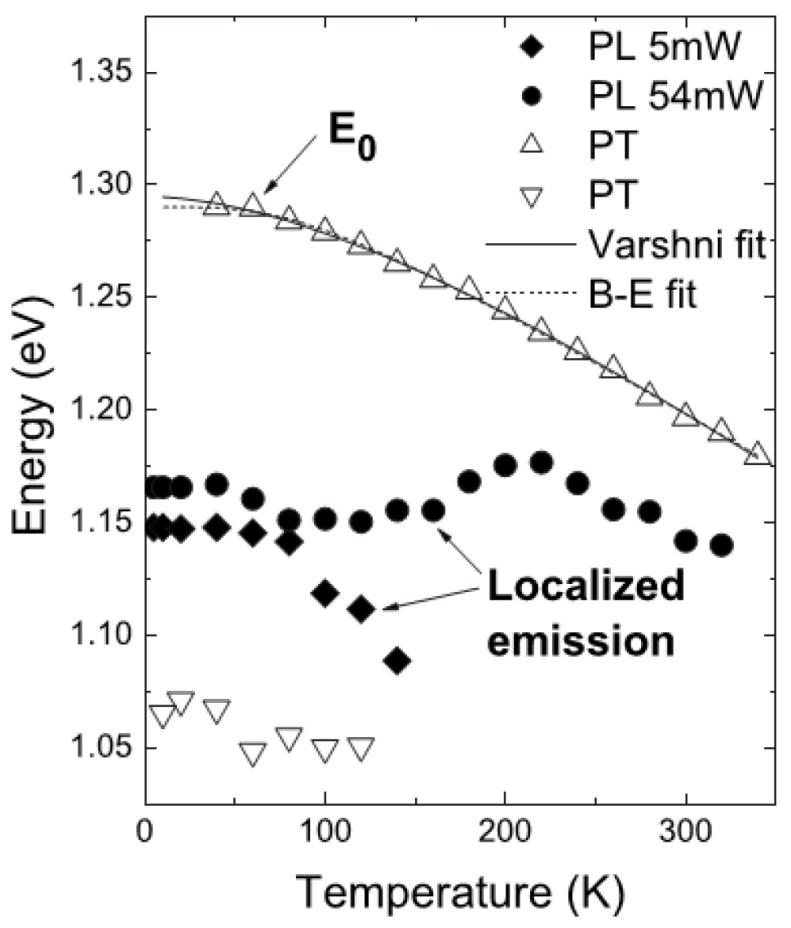

Figure 7. Temperature dependence of energy gap and $\mathrm{PL}$ peak energy for GaBiAs layer grown on (001) GaAs substrate [7]. bands below the energy gap suggests that at least two significantly different localization potentials or localization mechanisms could exist in this material.

Localization effects were also investigated by time-resolved photoluminescence measurements [7] (Fig. 8). An increase in the decay time with decreasing emission energy was observed, which provides evidence of a localized PL band. The decay time has been found to increase from 0.35 to $0.7 \mathrm{~ns}$ with a decrease of the emission energy. These values are smaller than the radiative lifetime of the free excitons in GaAs (about $3 \mathrm{~ns}$ ). The observed low decay time is similar to that observed for free carrier recombination in GaAs-based materials with dominating nonradiative recombination centers via defects and/or impurities. For localized excitons the radiative lifetime should be longer than 3 ns. However, the obtained PL results for different laser powers suggest an excitonic character for the PL band. Therefore, the observed low temperature emission was associated with excitonic emission with an important contribution of nonradiative centers, which reduce the decay times and PL intensity with increasing temperature.

The activation energy obtained for the quenching of PL intensity was determined for higher and lower

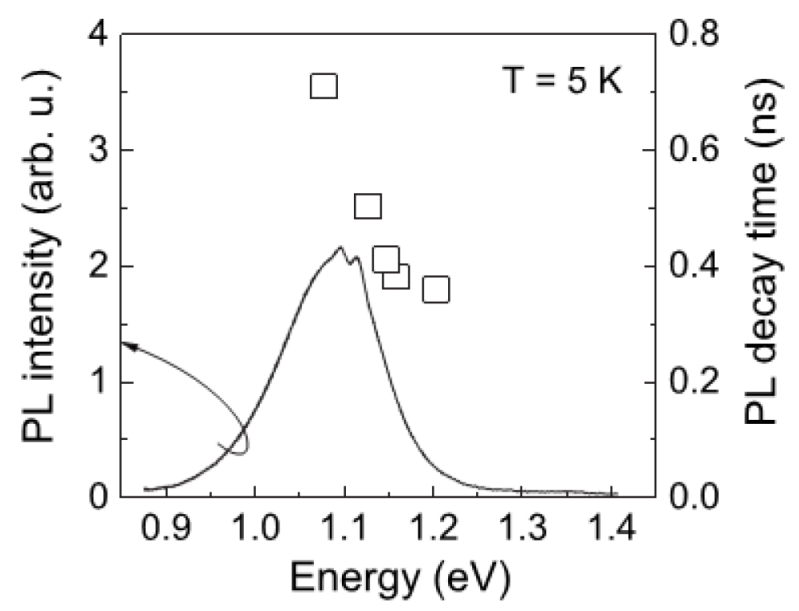

Figure 8. PL spectrum and PL decay time for different energies [7]. 
excitation laser power [7]. It was observed that for higher power the value is larger than that for lower excitation power but much smaller than the observed Stokes Shift [7]. This observation provides additional evidence of the presence of various localization potentials in this system. The high density of nonradiative centers in the layer is likely due to the MBE growth condition at low temperature $\left(350^{\circ} \mathrm{C}\right)$. In conclusion, carrier localization effects in GaBiAs layers was supported by different optical techniques [7].

(a)

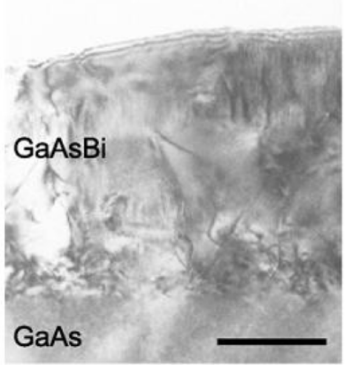

(d)

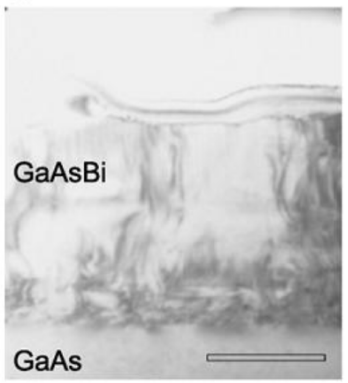

(e)

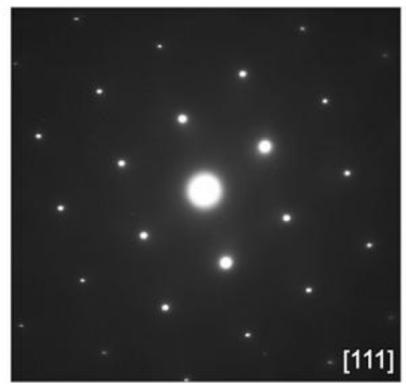

Figure 9. TEM images for as-grown (311)B sample (a, b) and for the annealed sample (c, d) [6].
Furthermore, it has been found that the nonradiative centers reduce the decay time and lead to important thermal quenching in the optical emission [7].

\section{Thermal Annealing Effects}

The effect of post-growth annealing on GaAsBi alloys grown on (311)B GaAs was analyzed by conventional transmission electron microscopy (TEM) (Fig. 9) [6]. Structural defects and a mosaic structure were observed in an as-grown GaAsBi layer [6]. Stacking
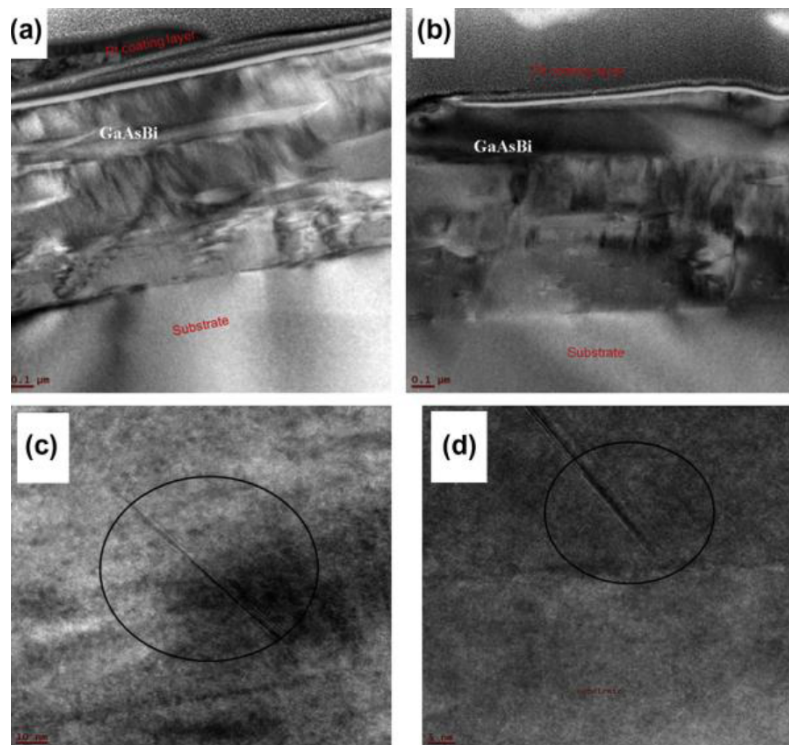

Figure 10. TEM Images of as-grown and annealed (001) GaBiAs layers (Sample Ms820) [8].
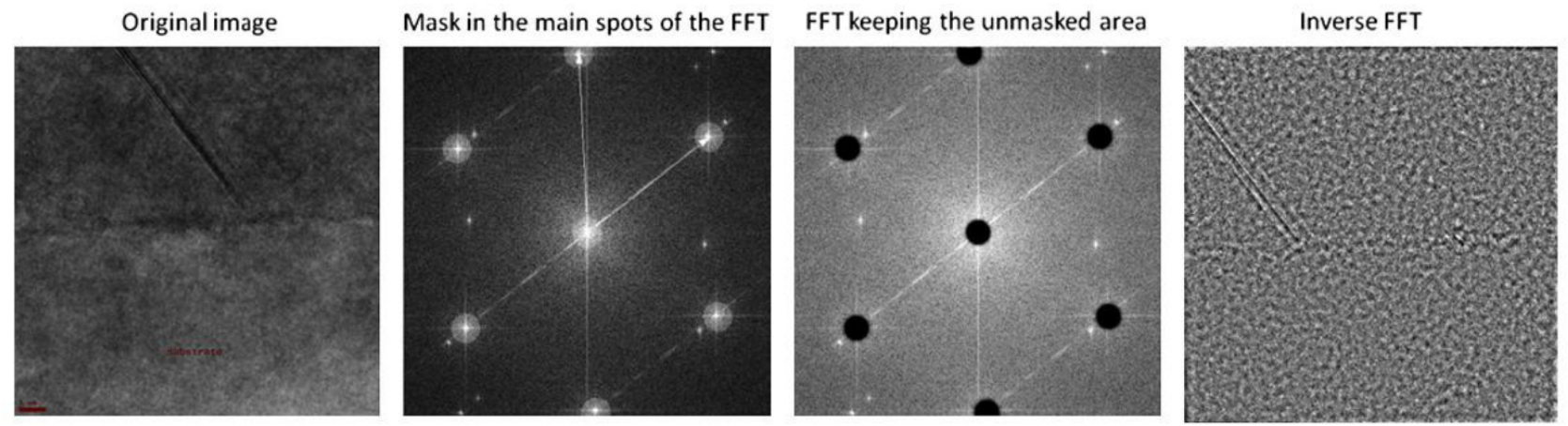

Figure 11. FFT analyses of TEM image for annealed sample [8]. 
faults were also observed around the GaAs/GaAsBi interface in high-resolution TEM images [6]. Long thermal annealing was performed for several samples at $473 \mathrm{~K}$ during 3 hours. It was observed that the layer is more homogeneous and that the mosaic structure disappears. The presence of defects appears to be consequently reduced, which results in an enhancement of emission intensity and narrowing of the optical linewidth [6]. The observed PL results are consistent with TEM results, which provide evidence of reduction of the nonradiative recombination centers after the long thermal annealing process.

GaBiAs samples grown on (001) GaAs substrates were also investigated [8]. TEM images (Figs. 10 and 11) for as-grown and annealed samples indicated the presence of important structural defects in the region close to the interface between GaAs and GaAsBi [8]. For the as-grown sample dark lines were observed along the growth directions, which could be related to threading dislocations. The observation of different contrast in the layer may indicate important changes in the composition [8]. The as-grown sample also showed the presence of stacking faults. Vertical contrast was observed after thermal annealing, and this may be associated with a possible modulation of the composition or stress in the layer. A Fast Fourier Transform (FFT) analysis was used to confirm the presence of stacking faults in these layers after thermal annealing [8].

The effect of thermal annealing on GaBiAs samples was also investigated by using a photoluminescence technique $[8,9]$. An important enhancement of the optical emission intensity with no significant changes in the PL peak position after long thermal annealing was observed (at $473 \mathrm{~K}$ during $3 \mathrm{~h}$ ) (Fig. 12 (a)). The temperature dependence of the PL energy peak was also investigated for both samples (as-grown and annealed). A pronounced S-shape behavior was observed, and this was associated with localization of carriers by alloy disorder, cluster formation, and po-
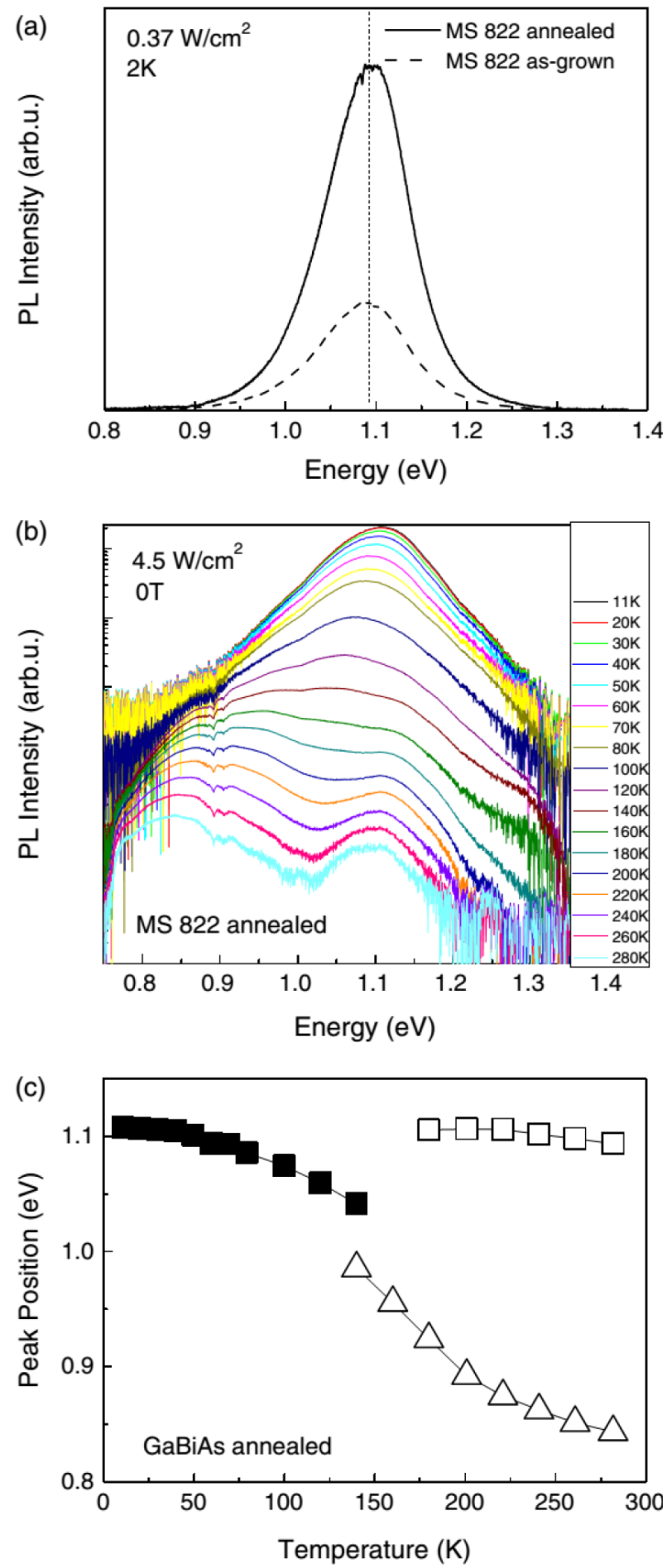

Figure 12. (a) PL spectra of as-grown and annealed (001) GaBiAs samples; (b) Temperature dependence of $\mathrm{PL}$ spectrum; (c) and peak position [9]. 
tential fluctuation $[8,9]$. As mentioned earlier, different emission peaks below the energy gap were observed and they are associated with the presence of defects of a different nature in the layer (Figs. 12 (b) and (c)).

\section{Magneto-Optical Properties}

As mentioned earlier, the incorporation of $\mathrm{Bi}$ in GaAs results in an important enhancement of the spin-orbit (SO) splitting energy, which is very interesting for spintronics applications. It was observed previously that the electron $g$-factor increases when a
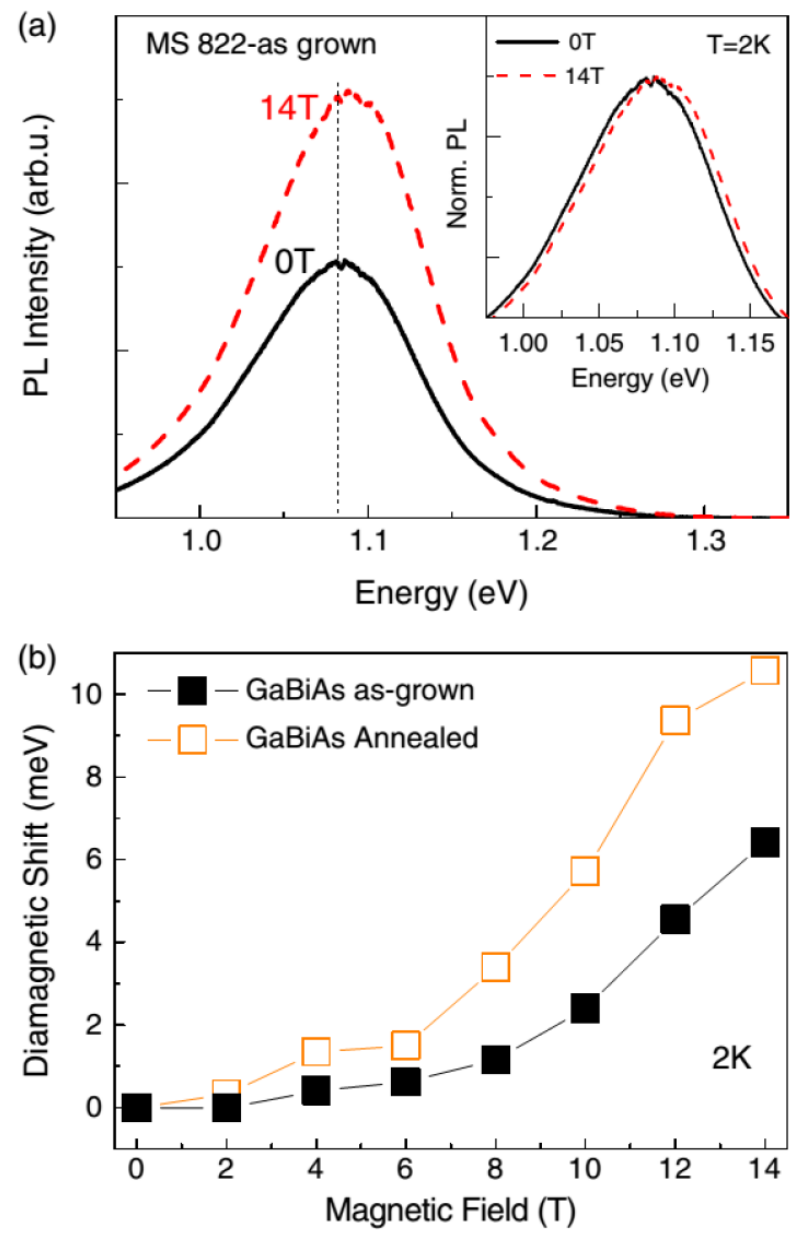

Figure 13. Magneto-PL spectra (a) and diamagnetic shift versus magnetic field at $2 \mathrm{~K}$ (b). (Sample Ms 820) [9]. small fraction of $\mathrm{Bi}$ is incorporated in GaAs [15]. In addition, it was reported that the spin relaxation times decrease, which is consistent with enhancement of the spin-orbit splitting energy [14,15]. Magneto-photoluminescence and effects of thermal annealing on the properties were investigated (Fig. 13) under high magnetic fields up to 14T [9]. For both samples (annealed and as-grown) a very small diamagnetic shift at low temperatures was observed and this was associated
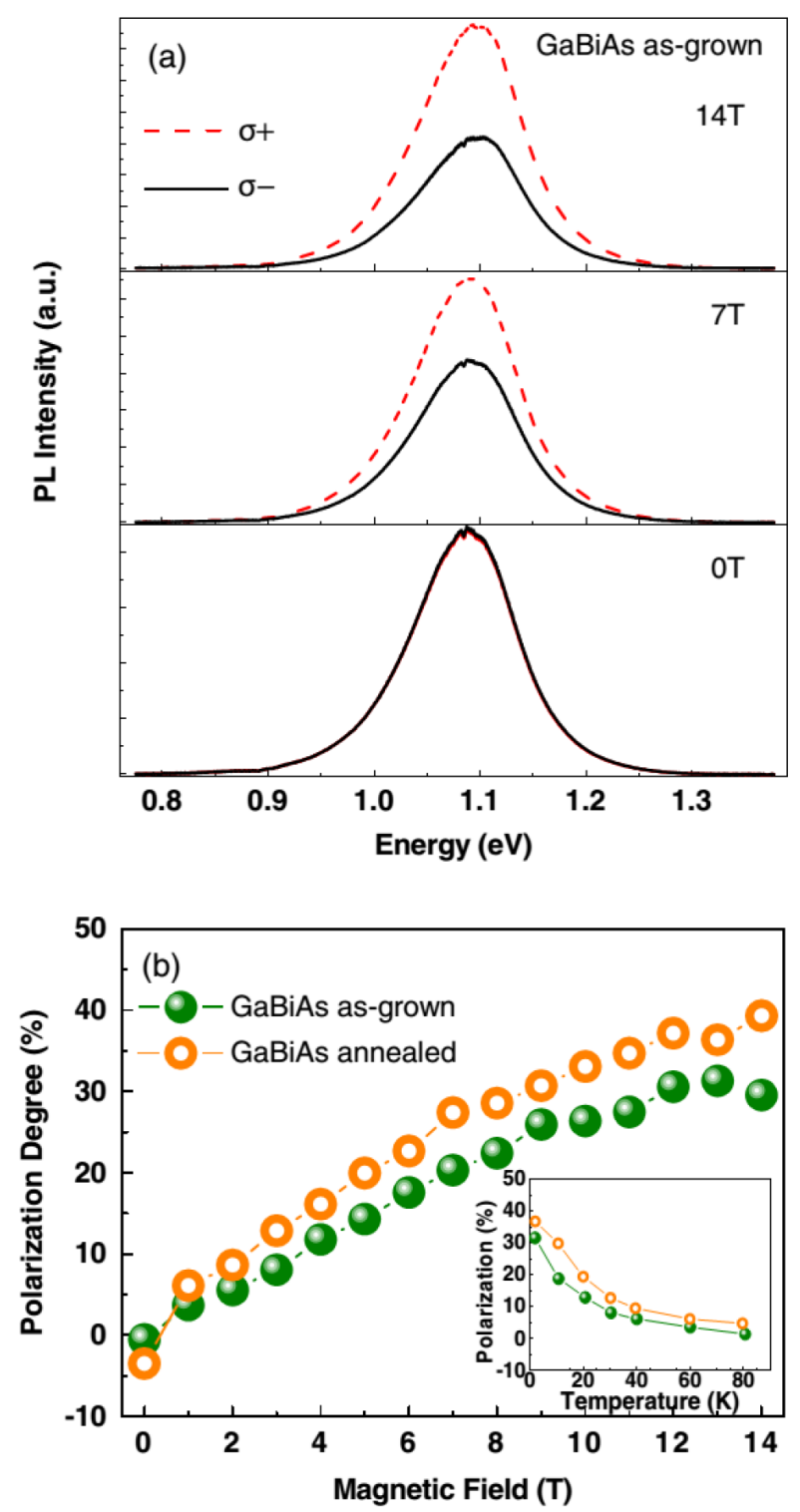

Figure 14. Polarization resolved Magneto-PL spectra (a) and Polarization degree versus magnetic field and temperature (b) [9]. 
mainly with localization of carriers by the presence of a high density of defects. It was observed that the diamagnetic shift increases after thermal annealing, which indicates a reduction of localization of carriers due to the reduction of defects in the layer.

Polarization resolved magneto-PL at low temperatures for GaBiAs layers was also investigated (Fig. 14 (a)). Spin polarization of electrons was estimated from circularly polarized $\sigma^{+}$and $\sigma^{-}$emissions. An important increase in the polarization degree in comparison to GaAs was observed, and this was likely due to $\mathrm{Bi}$-related changes in the band structure of GaBiAs. It was also found that the thermal annealing caused an increase of the polarization degree of electrons. A polarization degree up $41 \%$ was observed at low temperatures and under $14 \mathrm{~T}$. This increase of the electron spin polarization after thermal annealing was associated with a possible increase of spin relaxation times due to the reduction of defects in the material.

Furthermore, the polarization degree was found to depend on the laser intensity. In general, the polarization degree decreases with an increase of laser intensity. This effect is still under investigation.

In conclusion, important values of electron polarization degree in GaBiAs layers under high magnetic fields were observed. The increase of the spin polarization degree in comparison to GaAs was attributed to Bi-related changes in the band structure of GaBiAs. Furthermore, an enhancement of the optical emission intensity and the circularly polarization degree was observed after the thermal annealing process, and this was ascribed to a reduction of the density of defects after thermal annealing.

\section{Conclusion}

In this paper, we reviewed optical and structural studies performed on GaBixAs1-x epilayers grown by MBE on (311)B and (001) GaAs substrates with different
As fluxes. It was observed that under near-stoichiometric conditions the bismuth incorporation is higher for samples grown on (311)B GaAs substrates than for those grown on (001) substrates, and carrier localization effects in GaBiAs layers are clearly indicated for both samples by optical measurements. A higher density of defects was observed for (311)B samples. It has also been found that the nonradiative centers play a significant role in the recombination process in this material system. A significant improvement of the optical and spin properties was achieved after thermal annealing due to a reduction of defects in the GaBiAs layers. The results reported in this review show that the GaAsBi material system requires further optimization of the growth conditions in order to improve the material qualities for future technological applications. Finally, remarkable properties were observed for emerging GaBiAs alloys, and on this basis they are promising materials for several applications in spintronics and for long-wavelength optoelectronics.

\section{Acknowledgements}

The authors acknowledge financial support from the Brazilian Agencies [FAPESP (grant number 2012/24055-6), CAPES (PVE grant A067/2013), CNPq, and the UK Engineering and Physical Sciences Research Council.

\section{References}

[1] M. Henini, J. Ibanez, M. Schmidbauer, M. Shafi, S. V. Novikov, L. Turyanska, S. I. Molina, D. L. Sales, M. F. Chisholm, and J. Misiewicz, Appl. Phys. Lett. 91, 251909 (2007).

[2] B. Fluegel, S. Francoeur, A. Mascarenhas, S. Tixier, E. C. Young, and T. Tiedje, Phys. Rev. Lett. 97, 067205 (2006). 
[3] S. Francoeur, M. J. Seong, A. Mascarenhas, S. Tixier, M. Adamcyk, and T. Tiedje, Appl. Phys. Lett. 82, 3874 (2003).

[4] K. Oe, Jpn. J. Appl. Phys. 41, 2801 (2002).

[5] R. Kudrawiec, P. Poloczek, J. Misiewicz, M. Shafi, J. Ibanez, R. H. Mari, M. Henini, M. Schmidbauer, S. V. Novikov, L. Turyanska, S. I. Molina, D. L. Sales, and M. F. Chisholm, Microelectronics Journal 40, 537 (2009).

[6] J. F. Rodrigo, D. L. Sales, M. Shafi, M. Henini, L. Turyanska, S. Novikov, and S. I. Molina, Applied Surface Science 256, 5688 (2010).

[7] R. Kudrawiec, M. Syperek, P. Poloczek, J. Misiewicz, R. H. Mari, M. Shafi, M. Henini, Y. Galvão Gobato, S. V. Novikov, J. Ibáñez, M. Schmidbauer, and S. I. Molina, J. Appl. Phys. 106, 023518 (2009).

[8] O. M. Lemine, A. Alkaoud, H. V. Avanço Galeti, V. Orsi Gordo, Y. Galvão Gobato, Houcine Bouzid, A. Hajry, and M. Henini, Superlattices and Microstructures 65, 48 (2014).

[9] A. R. H. Carvalho, V. Orsi Gordo, H. V. A. Galeti, Y. Galvão Gobato, M. P. F. de Godoy, R. Kudrawiec, O. M. Lemine, and M. Henini, J. Phys. D: Appl. Phys. 47, 075103 (2014).

[10] A. R. Mohmad, F. Bastiman, C. J. Hunter, R.
Richards, S. J. Sweeney, J. S. Ng, and J. P. R. David, Appl. Phys. Lett. 101, 012106 (2012).

[11] A. R. Mohmad, F. Bastiman, C. J. Hunter, J. S. Ng, S. J. Sweeney, and J. P. R. David, Appl. Phys. Lett. 99, 042107 (2011).

[12] A. J. Ptak, France R., D. A. Beaton, K. Alberi, J. Simon, A. Mascarenhas, and C. S. J. Jiang, Cryst. Growth 338, 107 (2012).

[13] S. J. Sweeney and S. R. Jin, J. Appl. Phys. 113, 043110 (2013).

[14] B. Pursley, M. Luengo-Kovac, G. Vardar, R. S. Goldman, and V. Sih, Appl. Phys. Lett. 102, 022420 (2013).

[15] S. Mazzucato et al, Appl. Phys. Lett. 102, 252107 (2013).

[16] S. Mazzucato et al, Semicond. Sci. Technol. 28, 022001 (2013).

[17] H. Tong, X. Marie, and M. W. Wu, J. Appl. Phys. 112, 063701 (2012).

[18] G. Ciatto, E. C. Young, F. Glas, J. Chen, R. Alonso Mori and T. Tiedje, Phys. Rev. B 78, 035325 (2008).

[19] G. Ciatto, M. Thomasset, F. Glas, X. Lu, and T. Tiedje, Phys. Rev. B 82, 201304 (2010).

[20] D. L. Sales et al, Appl. Phys. Lett. 98, 101902 (2011). 